\title{
Small-scale heating events in the solar atmosphere
}

\section{Lifetime, total energy, and magnetic properties}

\author{
N. Guerreiro ${ }^{1}$, M. Haberreiter ${ }^{1}$, V. Hansteen ${ }^{2}$, and W. Schmutz ${ }^{1}$ \\ 1 Physikalisch-Meteorologisches Observatorium Davos, World Radiation Center, 7260 Davos Dorf, Switzerland \\ e-mail: nuno.guerreiro@pmodwrc.ch \\ 2 Institute of Theoretical Astrophysics, University of Oslo, PO Box 1029 Blindern, 0315 Oslo, Norway
}

Received 27 September 2016 / Accepted 4 April 2017

\begin{abstract}
Context. Small-scale heating events (SSHEs) are believed to play a fundamental role in understanding the process responsible for heating of the solar corona, the pervading redshifts in the transition region, and the acceleration of spicules.

Aims. We determine the properties of the SSHEs and the atmospheric response to them in 3D magnetohydrodynamics (3D-MHD) simulations of the solar atmosphere.

Methods. We developed a method for identifying and following SSHEs over their lifetime, and applied it to two simulation models We identified the locations where the energy dissipation is greatest inside the SSHEs volume, and we traced the SSHEs by following the spatial and temporal evolution of the maximum energy dissipation inside the SSHEs volume.

Results. The method is effective in following the SSHEs. We can determine their lifetime, total energy, and properties of the plasma, as well as the magnetic field orientation in the vicinity of the SSHEs.

Conclusions. We determine that the SSHEs that have the potential to heat the corona live less than 4 min, and typically the energy they release ranges from $10^{20}$ to $10^{24} \mathrm{erg}$. In addition, the directional change of the field lines on the two sides of the current sheet constituting the SSHEs ranges from $5^{\circ}$ to $15^{\circ}$ at the moment of the absolute maximum energy dissipation.
\end{abstract}

Key words. Sun: atmosphere - Sun: corona - Sun: flares

\section{Introduction}

A small-scale heating event (SSHE) is an impulsive energy release in the solar atmosphere in the $10^{17}-10^{27}$ erg range mainly driven by braiding of the magnetic field, as already discussed by Gold (1964) and Parker (1972). In recent decades, mounting evidence has emerged that SSHEs pervade the solar atmosphere (Lin et al. 1984; Porter et al. 1984, 1987; Krucker \& Benz 1998; Berghmans et al. 1998; Parnell \& Jupp 2000; Aschwanden et al. 2000, 2016; Hannah et al. 2008; Ramesh et al. 2013; Wang et al. 2015). However, the properties and influence of the SSHEs on the structure of the atmosphere are still not fully understood.

Parker (1988), driven by the discovery of SSHEs in X-rays by Lin et al. (1984), suggested that SSHEs could play a significant role in heating the solar corona. Since then, this concept has been followed up with increasing interest (Cargill 1994; Klimchuk \& Cargill 2001; Priest et al. 2002; Cargill \& Klimchuk 2004; Klimchuk 2006, 2015; Tomczyk et al. 2007; Parnell \& De Moortel 2012). Today, SSHEs are not only considered one of the most compelling mechanisms to explain coronal heating (Klimchuk 2006; Reale 2010), but they are also thought to play a significant role in explaining the acceleration of spicules (De Pontieu et al. 2007) and the origin of the pervading redshifts observed in the lower transition region (Doschek et al. 1976; Gebbie et al. 1981; Dere 1982; Klimchuk 1987; Rottman et al. 1990; Hansteen 1993; Brekke 1993; Achour et al. 1995; Brekke et al. 1997; Peter \& Judge 1999; Hansteen et al. 2010; Guerreiro et al. 2013b).

The observational study of SSHEs is challenging for several reasons: 1) many SSHEs are believed to have a spatial scale that lies below the currently available instrumental resolution
(Klimchuk 2015); 2) SSHEs can occur in regions of very low density, in which the plasma response is too faint to be detected by any observational instrument available today; 3) SSHEs can occur in regions where the thermal conduction is very efficient, which quickly erases the spatial information of the SSHEs; and 4) there are time delays between the occurrence of the SSHEs and the creation of ions that can emit the expected signatures, which can lead to false detections or detections that do not correspond to a single event alone. These aspects explain the difficulty in understanding the properties and ultimately the role of SSHEs in coronal heating, the driving of spicules, or the pervading redshift in the transition region.

In recent decades, studies have been conducted using 1D hydrodynamic simulations to explore the potential of SSHEs to explain the acceleration of spicules (Sterling 2000; Guerreiro et al. 2013a), but definitive conclusions have not been reached. At the same time, with the advent of simulations of the braiding process of the magnetic field (Galsgaard \& Nordlund 1996; Hendrix et al. 1996; Browning et al. 2008; Bowness et al. 2013) and $3 \mathrm{D}$ magnetohydrodynamics (3D-MHD) models of the solar atmosphere that span from the top of the convection zone to the corona (Gudiksen \& Nordlund 2005; Hansteen et al. 2007; Bingert \& Peter 2011), it has been consistently shown that SSHEs can heat the corona (Bingert \& Peter 2013; Guerreiro et al. 2015) and play a significant role in explaining the pervading redshifts in the transition region (Hansteen et al. 2010; Guerreiro et al. 2013b).

Even though significant progress has been made in understanding the implications of SSHEs for the solar atmosphere, some of their specific properties remain unknown or poorly 
understood. In this paper, we focus on studying the properties of the SSHEs in 3D-MHD simulations by extending the study by Guerreiro et al. (2015, hereafter Paper I), in which a method was developed to analyze SSHEs in 3D-MHD simulations at selected snapshots. Here, we go one step further and present a new method for following the SSHEs in time. The application of this method to 3D-MHD models with different resolutions opens the possibility of studying in detail some quantities that are paramount to better understanding SSHEs, such as their lifetimes, the total energy dissipated by each SSHE over its lifetime, the magnetic field, and the plasma properties in the vicinity of the SSHEs, among others.

This paper is organized as follows. A description of the models used in the study presented here is given in Sect. 2. The description of the method we developed to track the SSHEs in the 3D-MHD models is presented in Sect. 3. The study of the lifetime, total energy, magnetic properties, and the potential of the method to study the plasma response to the SSHEs are discussed in Sect. 4. Finally, the conclusions are given in Sect. 5.

\section{Models}

We used two 3D-MHD models of the quiet Sun atmosphere with different resolutions and magnetic topologies produced by the BIFROST code (Gudiksen et al. 2011). We refer to the models as the low-resolution model (LRM) and the high-resolution model (HRM). The LRM has a grid of $256 \times 128 \times 160$ points which is equivalent to a volume in the Sun of $16 \times 8 \times 16 \mathrm{Mm}^{3}$, and the HRM has a grid of $768 \times 768 \times 768$ points, equivalent to a volume in the Sun of $24 \times 24 \times 16 \mathrm{Mm}^{3}$. In both models the grid is uniformly spaced in the $x$ - and $y$-directions and non-uniformly spaced in the $z$-direction. The spatial resolution is $65 \mathrm{~km}$ and $31 \mathrm{~km}$ in the $x$ - and $y$-direction for the LRM and HRM, respectively. In the $z$-direction, the maximum resolution is $32 \mathrm{~km}$ and $12 \mathrm{~km}$ for the LRM and HRM, respectively. The zero point for the height scale, $z=0 \mathrm{~km}$, is defined as the point where the optical depth is $\tau_{500}=1$.

Upon initiation of the runs, the LRM ran for $1 \mathrm{~h}$ solar time and the HRM for 50 min solar time. The snapshots, which represent the state of the simulation at varying instants, have a cadence of $10 \mathrm{~s}$ for both models. Similar models to those used here have been extensively described in the literature. For the LRM see, e.g., Paper I and references therein and for the HRM see, e.g., Hansteen et al. (2015) where a similar model is described in detail.

In summary, the models span from the top of the convection zone to the corona. The convection is achieved and maintained by setting the entropy of the inflow of material at the bottom of the simulation box and solving the equations of radiative transfer in four frequency bins such that an atmosphere with an effective temperature of approximately $T_{\text {eff }} \sim 5800 \mathrm{~K}$ is formed (Nordlund 1982; Steffen \& Muchmore 1988). The models include artificial viscous diffusivity and magnetic resistivity which are responsible for the magnetic and viscous heating on small scales. A comprehensive description of how these were implemented is given in Paper I and references therein. Essentially, as the magnetic field is stressed by the convective motions, gradients increase and lead to the formation of current sheets (Galsgaard \& Nordlund 1996) and dissipation which is responsible for the heating. The current sheets form finger-like structures (Hansteen et al. 2015) that subsequently collapse to the dimensions of a few grid cells. This scaling results from the functional form of the resistivity which is proportional to the size of the grid, where the grid Reynolds numbers are slightly greater than those required to resolve the current sheets in a certain grid. It is clear that in the Sun, the current sheets are much thinner that what can be resolved by the simulations. However, as shown by several authors, e.g., Galsgaard \& Nordlund (1996) or Gudiksen \& Nordlund (2005), the total dissipation in a certain current sheet is independent of the resolution of the simulation, but the scale on which the dissipation occurs changes with the resolution. Even if the resolution of the simulation models plays an important role in the scale on which the dissipation occurs, it is clear that studying the small scales in the models can give good insight into the properties of the SSHEs. In the photosphere, a non-gray, non-LTE radiative transfer is used, and in the chromosphere the radiative transfer is considered to be optically and effectively thin. The treatment of radiation in the chromosphere was conducted using the description given by Hayek et al. (2010) and Carlsson \& Leenaarts (2012). The equation of state was computed as described by Gudiksen et al. (2011). The thermal conduction along the magnetic field is included and is implemented using a multi-grid method. In the models, the boundaries are periodic in the $x$ - and $y$-directions, and non-periodic in the $z$-direction. The upper boundary is transparent to waves using characteristic boundary conditions and the lower boundary allows flows to exit without constraints.

Figure 1 displays the typical Joule heating $\left(\eta J^{2}\right)$ distribution over time for the LRM (top panel) and for the HRM (bottom panel) against height at two instants in time, respectively. The darker regions correspond to a higher concentration of data points, the brighter regions correspond to a lower concentration, and white represents the absence of data points. The white dashed line indicates the average of the $\eta J^{2}$ against height. For the LRM, the data correspond to the state of the simulation at $2000 \mathrm{~s}$ solar time. At this instant, $\eta J^{2}$ decreases exponentially above $1.5 \mathrm{Mm}$ and has a scale height of about $1300 \mathrm{~km}$. Above $1.5 \mathrm{Mm}$, the scatter in the strength of $\eta J^{2}$ ranges from roughly 8 orders of magnitude at the bottom of the chromosphere to 5 orders of magnitude in the corona. The HRM at $600 \mathrm{~s}$ shows similar behavior to the LRM. The $\eta J^{2}$ decreases exponentially roughly above $1.5 \mathrm{Mm}$, but this model has a larger scale height, on the order of $1600 \mathrm{~km}$. The variability of $\eta J^{2}$ in this model is roughly 8 orders of magnitude at any given height above $1.5 \mathrm{Mm}$. We can see that the scale height is different in the two models, which results from the different topologies of the magnetic field and is comprehensively explained in Hansteen et al. (2015).

\section{Tracking of the SSHEs in 3D-MHD simulations}

In Paper I, we introduced a method for identifying and selecting SSHEs in 3D-MHD simulations at specific instants in time. In short, we identify SSHEs by determining the local maxima of the Joule heating $\left(\eta J_{\max }^{2}\right)$ or the Joule heating per unit mass $\left(\eta J^{2} / \rho\right)$. Here, we build on this work and present a technique for following SSHEs over time. Essentially, we choose a snapshot and identify the SSHEs in it, which are at different stages of their evolution, and track them forward and backward in time focusing on the SSHEs that present a clear rising and decaying phase.

As described in Paper I, the underlying assumption used to identify SSHEs is based on the principle that every SSHE has a local maximum of energy dissipation in its volume independent of its evolutionary state. The local maxima of SSHEs can be identified in the different snapshots, as described in Paper I. The challenge, however, is to find which local maxima in consecutive 
N. Guerreiro et al.: Small-scale heating events in the solar atmosphere. II.
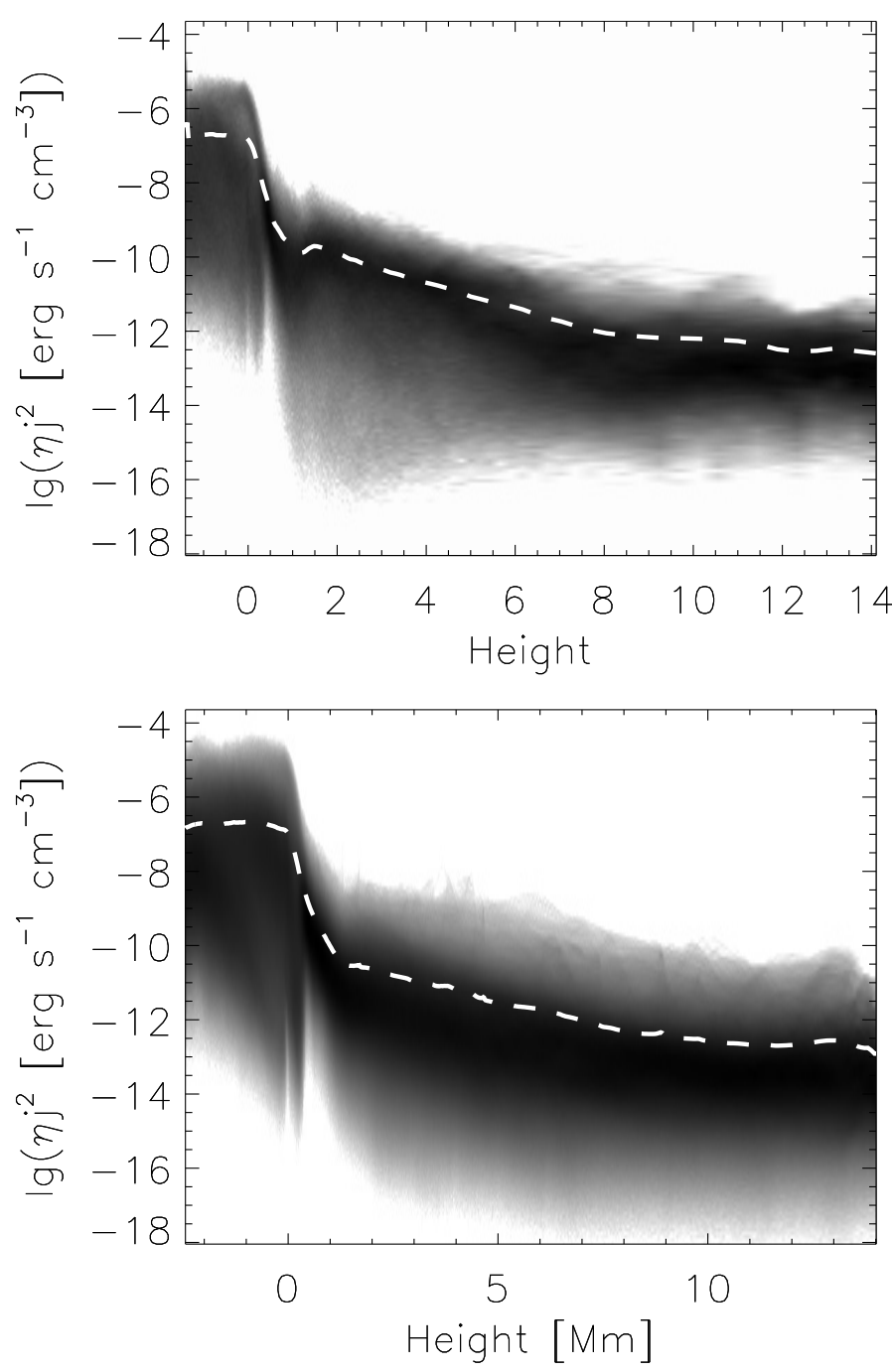

Fig. 1. Top panel: Joule heating $\left(\eta J^{2}\right)$ at $t=2000 \mathrm{~s}$ as a function of height for the LRM. Bottom panel: Joule heating $\left(\eta J^{2}\right)$ at $t=600 \mathrm{~s}$ as a function of height for the HRM. In both panels the dark regions stand for high concentration of data points, gray regions for low concentration, and white for the absence of data points. The white dashed lines give the average $\eta J^{2}$ as a function of height.

snapshots correspond to the same SSHE at its different evolutionary states.

Formally, we consider two consecutive snapshots, snapshot $s_{j}$ and snapshot $s_{j+1}$, each with a total of $N_{j}$ and $N_{j+1}$ SSHEs. The challenge in studying the evolution of the SSHE $i$ in snapshot $s_{j}$ is to identify the local maximum of energy dissipation $\left(\eta J_{\max }^{2}\right)$ in snapshot $s_{j+1}$ that corresponds to the previous or next evolutionary state of the SSHE $i$, depending on whether we are following the SSHE forward or backward in time. It is important to note that the assumption here is that by following the evolution of the local maximum of a SSHE, we are following the SSHE itself. In order to establish the connection between the different local maxima in consecutive snapshots, we start by selecting two consecutive snapshots $s_{j}$ and $s_{j+1}$. The snapshot $s_{j}$ is defined as the control snapshot and $s_{j+1}$ as the test snapshot. These should not be the last 15 snapshots of the simulated data because in this case it is not possible to track the SSHEs over their entire life span.

The method starts by identifying the location of a local maximum (a SSHE) in $s_{j+1}$, then it determines in $s_{j}$ whether there is a local maximum in a pre-determined search volume, neighboring the location of the local maximum in $s_{j+1}$. If there are local maxima in the search volume, a link is established according to the following situations that can occur: (1) if there is only one local maximum inside the search volume in $s_{j}$, we consider the local maximum to be related to that of $s_{j+1}$; (2) if there is more than one local maximum in the search volume in $s_{j}$, the local maximum with the closest value to the local maximum value in $s_{j+1}$ is assumed to be related to the local maximum in that snapshot. We note that we do not have a physical reason to justify the link between the local maxima based on the closest value, hence it is clear that this could lead to false positives. Nevertheless, in practice the choice seems to be reasonable because of the constraints described in the next paragraph which help to remove the false positives; and (3) if there is no local maximum, the local maximum in $s_{j+1}$ is disregarded from the set of SSHEs tracked. This procedure is applied to all local maxima in $s_{j+1}$ that are above the background. When all the SSHEs in $s_{j+1}$ have been compared to those in $s_{j}$, then $s_{j+1}$ becomes $s_{j}$ and the next snapshot in the data sequence, i.e., $s_{j+2}$ becomes $s_{j+1}$. The new $s_{j}$ differs from the previous $s_{j+1}$ because all the SSHEs (local maxima) that did not have a corresponding local maximum from the $s_{j+1}$ to the initial $s_{j}$ are disregarded. Therefore, the new $s_{j}$ only contains the SSHEs that have a corresponding local maximum in the previous $s_{j}$. We apply the same methodology to determine the corresponding local maxima in the two snapshots currently being analyzed, but additional considerations in relation to their previous evolution are made as discussed below.

When we follow SSHEs forward in time starting at snapshot $s_{j}$ at a certain instant $t$, and assuming that the SSHEs represent a rising and decaying phase, we apply the following conditions: (1) if the local maximum $\left(\eta J_{\max }^{2}(t+10)\right)$ in the first $s_{j+1}$ is smaller than the corresponding local maximum in the initial $s_{j}$ at instant $t$, we only accept a local maximum as part of the SSHEs in the new $s_{j+1}$ at $(t+20) \mathrm{s}$ if $\eta J_{\max }^{2}(t+10)>\eta J_{\max }^{2}(t+20)$; and (2) if the value of the local maximum in the first $s_{j+1}$ at $(t+10) \mathrm{s}$ is higher that the corresponding value in $s_{j}$ at $t$ we accept values for the SSHEs in the new $s_{j+1}$ at $(t+20) \mathrm{s}$ that can be higher or lower than the value of the local maximum in the first $s_{j+1}$ at $(t+10) \mathrm{s}$. These conditions guarantee that we do not follow unrealistic SSHEs and that they present a rising and decaying phase. We apply this method to consecutive snapshots until we stop finding local maxima in $s_{j+1}$ that can be related to $s_{j}$ or the value of the local maxima falls below the background. Similar conditions are applied when following the SSHEs backward in time. We have also run tests assuming small variations (scatter) in the rising and decaying phase, i.e., relaxing the assumption of a monotonic rising and decaying phase of the SSHEs. The influence of this consideration for the results presented here is negligible.

The search volume is different depending on the model we use. We define a search volume with a dimension of 27 cells $(195 \times 195 \times \sim 200) \mathrm{km}^{3}$ for the LRM, while a 125 cell volume $(155 \times 155 \times \sim 100) \mathrm{km}^{3}$ is used for the HRM. These choices of search volumes are related to the average plasma velocity in the models during the period of interest and to their resolution. We choose to analyze SSHEs for periods of time when the average velocity of the plasma in the upper regions of the atmosphere is between 10 and $15 \mathrm{~km} \mathrm{~s}^{-1}$. This implies that the majority of the SSHEs remain inside the search volume for periods on the order of $10 \mathrm{~s}$ or above. The search volume in the LRM is larger than the search volume in the HRM. Thus in the HRM, more SSHEs can travel outside the search volume and therefore the method 
does not allow us to follow them over their lifetime. However, as is shown later, the loss of some SSHEs have a negligible impact on the results of this work, in particular with regard to estimating the lifetime of the SSHEs.

The number of SSHEs detected depends on the background value we choose. We tracked the SSHEs considering two different backgrounds $10^{17}$ and $10^{18} \mathrm{erg} \mathrm{s}^{-1}$ in the two models. The choice of $10^{18} \mathrm{erg} \mathrm{s}^{-1}$ as a background value is based on the idea, that if the quiet corona can be heated by SSHEs, then the distribution of the energy of the SSHEs has to extend down to $10^{18}$ erg (Berghmans 2002) in order to balance the energy losses of the corona. The use of different backgrounds aims to determine the implications of the choice of background for the number of SSHEs computed. We select SSHEs that occur from the photosphere to the corona in both models. However, in the LRM, we analyzed the full simulation box, excluding only the region corresponding to the top of the convection zone. In the HRM, due to computational and time constraints, we only analyzed a subvolume of the full computational box. The region selected is equivalent to $24 \times 4 \times 14 \mathrm{Mm}^{3}$, which corresponds to the cell range [0:768, 320:447, 158:768].

The top panel in Fig. 2 displays the temporal evolution of a set of SSHEs that illustrates the type of SSHEs selected and those dismissed in the work presented here. The figure shows six SSHEs taken from the LRM. The SSHEs considered in the overall statistics are similar to $\mathrm{SSHE}_{\mathrm{a}}, \mathrm{SSHE}_{\mathrm{b}}$ and $\mathrm{SSHE}_{\mathrm{c}}$. These SSHEs show a clear rising and decaying phase in the energy release at the local maximum $\left(\eta J_{\max }^{2}\right)$. The rising and decaying phases are not necessarily symmetrical or have a similar number of points in the rising and decaying phase. The SSHEs dismissed are those that do not have a clear rising and decaying phase or SSHEs that consist only of two data points. Examples of the disregarded SSHEs are also shown in Fig. 2 and are identified as $\mathrm{SSHE}_{\mathrm{d}}, \mathrm{SSHE}_{\mathrm{e}}$, and $\mathrm{SSHE}_{\mathrm{f}}$. We noted that the method currently does not account for the SSHEs that split into different SSHEs. The SSHEs that do not show a clear rising and decaying phase like $\mathrm{SSHE}_{\mathrm{d}}$ and $\mathrm{SSHE}_{\mathrm{f}}$ can represent SSHEs that split. $\mathrm{SSHE}_{\mathrm{f}}$ represents an SSHE that potentially splits into two or several SSHEs with local maxima that have much smaller magnitudes, possibly with a value that is smaller than the background value, and therefore our method is not able to continue tracking it. A second explanation for the loss of an SSHE by the method is that the SSHE traveled outside the maximum volume prescribed to search for the SSHEs. For example, $\mathrm{SSHE}_{\mathrm{d}}$ was lost when it was tracked backward in time. It was probably lost by the method because it is a SSHE that, at a certain stage of its lifetime, traveled a distance that cannot be covered by the search region assigned in the method. This type of SSHE can also result from a SSHE that splits into several SSHEs and one of the other SSHEs have a local maximum of energy dissipation that is closer to the original value of the SSHE that it splits from. By construction, therefore, that SSHE would be associated with the original SSHE and the remaining ones would show a profile similar to that for $\mathrm{SSHE}_{\mathrm{d}}$. The SSHEs tracked where only two local maxima were associated with a SSHE similar to $\mathrm{SSHE}_{\mathrm{e}}$, for example, can have the same explanation as the cases above. Alternatively, these SSHE are above the background for a period shorter than $29 \mathrm{~s}$, during which period only two snapshots are taken.

A test performance of the method is shown in the bottom panel of Fig. 2 which shows the number of SSHEs with a clear rising and decaying phase for the LRM determined by the method inside each energy decade from $1 \times 10^{19}$ to $1 \times 10^{27} \mathrm{erg}$. The dashed line corresponds to the SSHEs detected when using
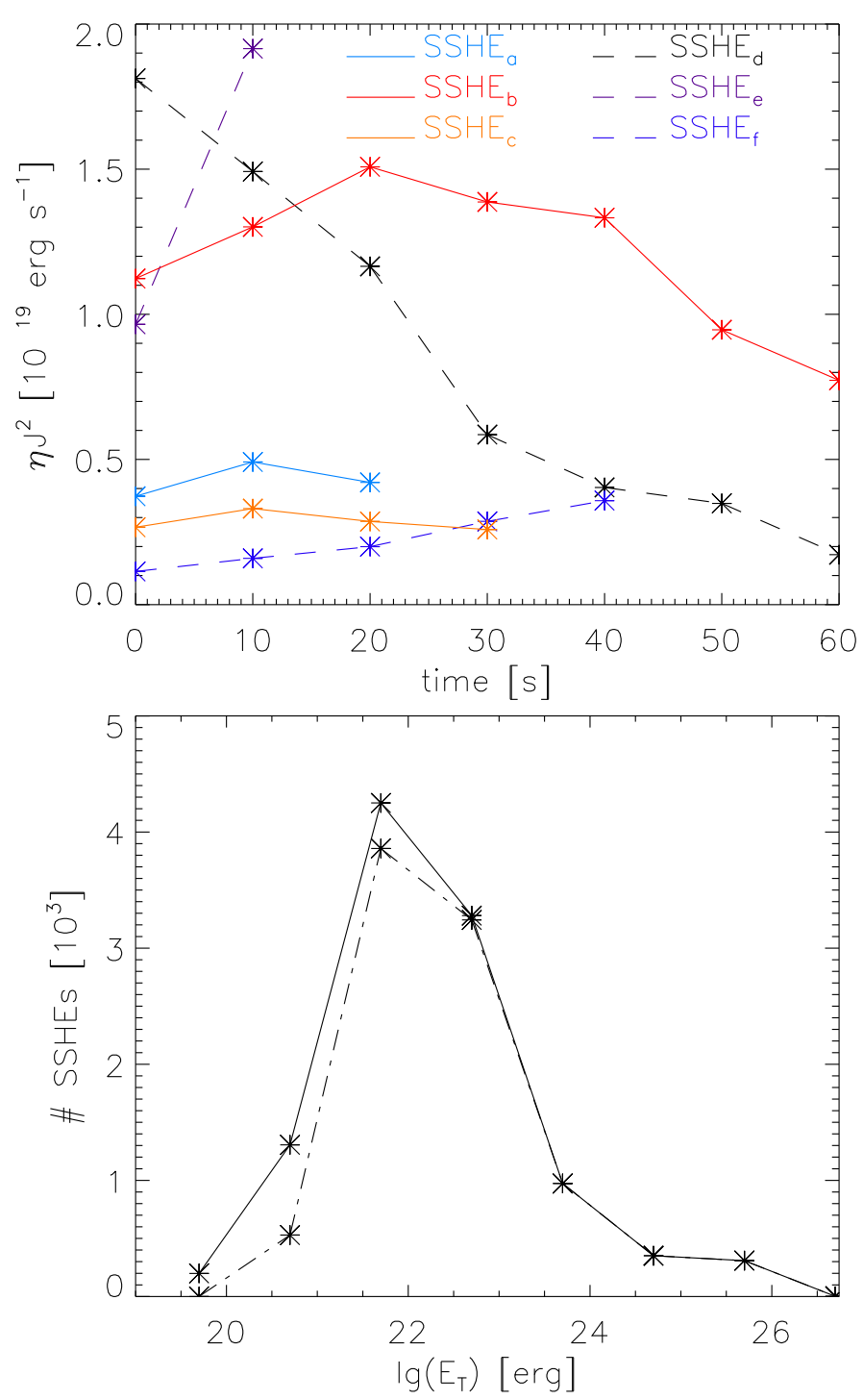

Fig. 2. Top panel: six SSHEs tracked by the method over their lifetime in the LRM. The data points show the value of the Joule heating $\left(\eta J^{2}\right)$ at the location (cell) of maximum dissipation of the SSHEs while they are above the background, which in this case is chosen as $10^{17} \mathrm{erg} \mathrm{s}^{-1}$. The data points connected by a solid line represent the SSHEs considered in the statistics while those connected by a dashed line represent the disregarded SSHEs. Bottom panel: Number of SSHEs with a clear rising and decaying phase inside each energy decade ranging from $1 \times 10^{19}$ to $1 \times 10^{27} \mathrm{erg}$ for two different backgrounds $10^{18} \mathrm{erg} \mathrm{s}^{-1}$ (dashed line) and $10^{17} \mathrm{erg} \mathrm{s}^{-1}$ (solid line) for the LRM.

the background $1 \times 10^{18} \mathrm{erg} \mathrm{s}^{-1}$ and the solid line corresponds to the SSHEs computed with the background $1 \times 10^{17} \mathrm{erg} \mathrm{s}^{-1}$. The plot shows that the method computes the SSHEs consistently when using the same search volume, but different backgrounds. The SSHEs with energy above $1 \times 10^{22}$ erg are consistently detected using the two different backgrounds. From $1 \times 10^{19}$ erg to $1 \times 10^{22}$ erg there is a difference in the SSHEs computed. This difference results from the larger number of SSHEs that are computed inside that energy interval when the background value is lowered. 
Table 1. Low resolution model (LRM), high resolution model (HRM), background $(\mathrm{Bg})$, total of SSHEs detected (\# SSHEs $\left.{ }^{\mathrm{T}}\right)$, SSHEs that present a clear rising and decaying phase (\# SSHEs ${ }^{\mathrm{S}}$ ), and average lifetime of the SSHEs $(\langle\mathrm{LT}\rangle)$.

\begin{tabular}{ccccc}
\hline \hline Model & $\begin{array}{c}\text { Bg }_{\left.\mathrm{erg} \mathrm{s}^{-1}\right]} \\
\text { \# SSHEs }^{\mathrm{T}}\end{array}$ & \# SSHEs $^{\mathrm{S}}$ & $\begin{array}{c}\langle\mathrm{LT}\rangle \\
{[\mathrm{s}]}\end{array}$ \\
\hline LRM & $10^{17}$ & 18689 & 10670 & $52.46 \pm 21.59$ \\
LRM & $10^{18}$ & 15738 & 9265 & $52.21 \pm 21.18$ \\
HRM & $10^{17}$ & 57829 & 36587 & $56.40 \pm 25.21$ \\
HRM & $10^{18}$ & 23088 & 15556 & $57.99 \pm 26.45$ \\
\hline
\end{tabular}

\section{Results and discussion}

\subsection{Lifetime}

The lifetime of a SSHE is defined as the period when the local maximum of the SSHE is above the background and the calculation of the average lifetime includes all the SSHEs that present a clear rising and decaying phase in their local maximum energy dissipation, i.e., $\eta J_{\max }^{2}(t)$. In the LRM, we started tracking the SSHEs $1.0 \times 10^{3} \mathrm{~s}$ after the beginning of the simulation and the method followed them forward and backward in time. In the HRM, we started tracking the SSHEs at $2.7 \times 10^{3} \mathrm{~s}$ after the beginning of the simulation and we followed the same procedure as for the LRM.

Table 1 and Fig. 3 give information about several parameters associated with the SSHE lifetimes computed in the two models for the different backgrounds $(\mathrm{Bg})$. The table lists the number of SSHEs followed for the different backgrounds (\# SSHEs ${ }^{\mathrm{T}}$ ), the number of SSHEs that have a distinct rising and decaying phase of the $\eta J_{\max }^{2}(t)$ local maximum (\# SSHEs ${ }^{\mathrm{S}}$ ), and their weighted average lifetime $(\langle\mathrm{LT}\rangle)$, which is given by the weighted average

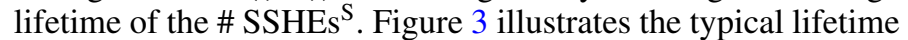
distribution of the SSHEs for the LRM (triangles) and the HRM (asterisks) for the $10^{17} \mathrm{erg} \mathrm{s}^{-1}$ background. In both cases the distribution is similar to a power law. The shortfall for the SSHEs with a lifetime of $30 \mathrm{~s}$ suggests a decrease in the number of shortlived SSHEs. However, we need models with a higher cadence to fully verify this tendency, which we currently do not have.

The different backgrounds naturally give a different number of tracked SSHEs. For the $10^{17}$ and $10^{18} \mathrm{erg} \mathrm{s}^{-1}$ backgrounds the method followed 18689 and 15738 SSHEs, respectively, for the LRM simulation. From these sets of SSHEs for the different backgrounds about $57 \%$ and $59 \%$ of the SSHEs, respectively, have a clear rising and decaying phase. In the HRM, the method tracked 57829 and 23088 SSHEs for the backgrounds $10^{17}$ and $10^{18} \mathrm{erg} \mathrm{s}^{-1}$, respectively. From these, $36587(63 \%)$ and 15556 $(67 \%)$ have a clear rising and decaying phase. The number of SSHEs detected is significantly higher than in the LRM, but the volume, resolution, and magnetic distribution is different in the two models. Therefore, we refrain from making comparisons between the number of SSHEs detected and selected in the two different models. Althought it is interesting to understand the relation between the magnetic field topology and the number of SSHEs occurring at each moment, we will leave that for a future study. The difference between \# SSHEs ${ }^{\mathrm{T}}$ and \# SSHEs $^{\mathrm{S}}$ corresponds to SSHEs that travel outside the search volume, and SSHEs that split.

For the LRM, the $\langle\mathrm{LT}\rangle$ of the SSHEs remains nearly independent of the choice of background and is about $52 \mathrm{~s}$. In the HRM case, the $\langle\mathrm{LT}\rangle$ ranges roughly from 56 to $58 \mathrm{~s}$ for the two backgrounds; it is shorter for the background with the lowest value by

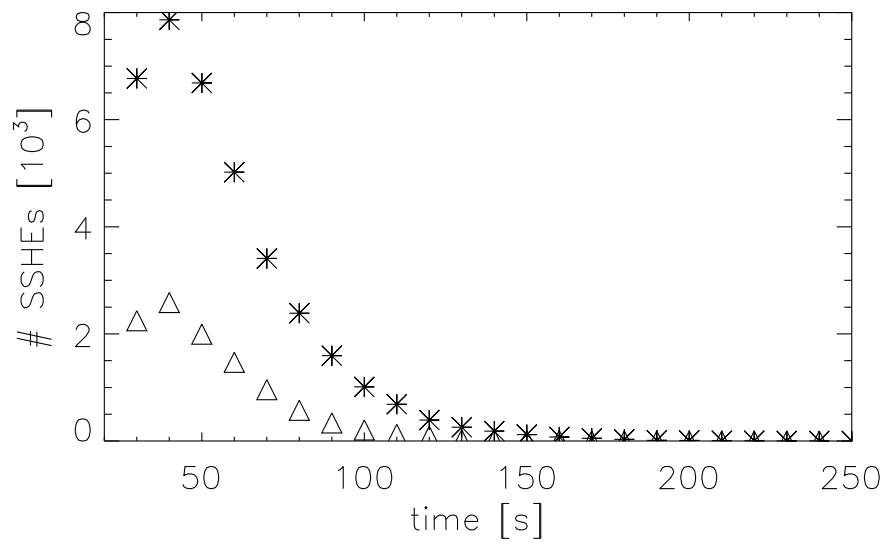

Fig. 3. Number of SSHEs vs. lifetime for the $10^{17} \mathrm{erg} \mathrm{s}^{-1}$ background. The triangles represent the SSHEs sample from the LRM, while the asterisks represent the sample from the HRM.

about two seconds, and the variation between the two values for the different backgrounds is higher than observed for the LRM. Nevertheless, the $\langle\mathrm{LT}\rangle$ for the backgrounds in the two models are well within the uncertainty range of each other. We note that the number of SSHEs tracked in the LRM is significantly greater than the number of SSHEs shown in Paper I because we consider all the local maxima associated with $\eta J^{2}$ here and not the local maximum given by the $\eta J^{2} / \rho$. The average lifetime of the SSHEs presented here is slightly lower than the value found by Hansteen et al. (2015) for this model. They define the lifetime of the SSHEs as the width at half-height of the auto-covariance function associated with each cell in the computational box, including those we regard as belonging to the background. The background cells generally vary little over short periods of time, and therefore have a larger width at half-height and consequently contribute to increasing the average lifetime of the SSHEs.

It is important to note that the temporal and spatial resolution of the models used is not sufficient to study the role of very short-lived SSHEs in coronal heating because we disregard the SSHEs shorter than $30 \mathrm{~s}$. We consider the average lifetime of the SSHEs studied in these two models as the expected lifetime of SSHEs when observing the Sun with resolutions similar to those of the models. On the other hand, Fig. 3 shows that the upper limit for the lifetime of the SSHEs that heat the corona in these models is $\sim 4 \mathrm{~min}$.

\subsection{Total energy}

The total energy $\left(E_{\mathrm{T}}\right)$ of the SSHEs is determined by integrating the instantaneous total energy dissipated $\left(E_{\mathrm{t}}\right)$ by the SSHEs every $10 \mathrm{~s}$ during their lifetime using the trapezoid rule. The value of the energy dissipated by a SSHE computed in the different snapshots is calculated after determining the volume of the SSHE, as was comprehensively described in Paper I: $E_{\mathrm{T}}=$ $\int_{t} \int_{V} \eta J^{2} \mathrm{~d} V \mathrm{~d} t$, where $t$ stands for the lifetime of the SSHE and $V$ stands for the volume of the SSHE at a certain instant.

Figure 4 displays the probability distribution of $E_{\mathrm{T}}$ in logarithmic scale versus the lifetime of the SSHEs. The left panels correspond to the LRM and the right panels to the HRM. The top panels represent the SSHEs selected with a background of $1 \times 10^{18} \mathrm{erg} \mathrm{s}^{-1}$ and the bottom panels with a background of $1 \times 10^{17} \mathrm{erg} \mathrm{s}^{-1}$. We can see that the choice of the background affects the lower cutoff of $E_{\mathrm{T}}$ of the SSHEs studied. The panels representing the SSHEs computed using the $1 \times 10^{18} \mathrm{erg} \mathrm{s}^{-1}$ 

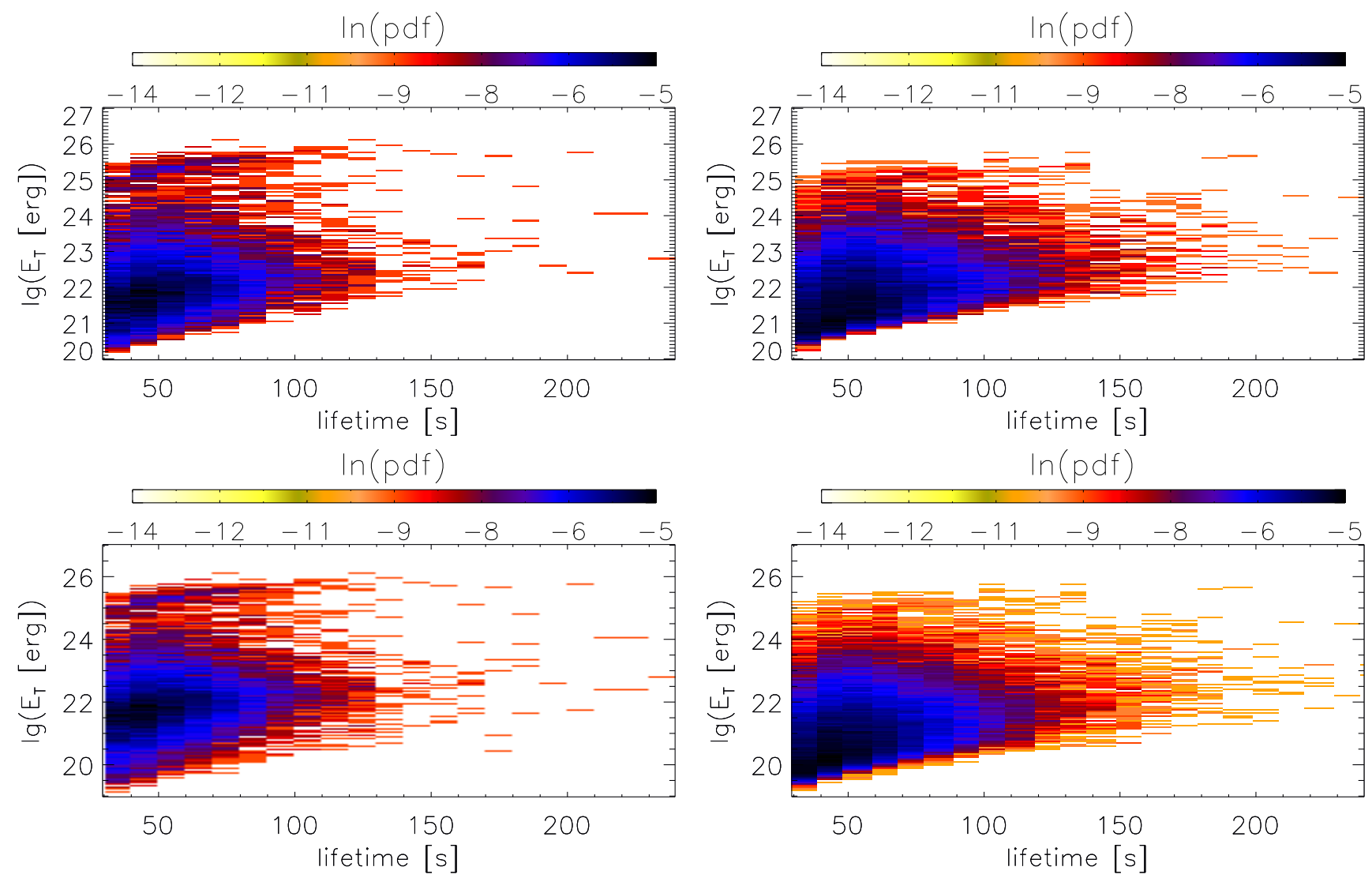

Fig. 4. Probability distribution function of the total energy $\left(E_{\mathrm{T}}\right)$ of the individual SSHEs vs. their lifetime considering SSHEs selected using different backgrounds for the LRM (left) and HRM (right). The backgrounds are $10^{18} \mathrm{erg} \mathrm{s}^{-1}$ and $10^{17} \mathrm{erg} \mathrm{s}^{-1}$ for the top and bottom panels, respectively. The darker regions correspond to a high concentration of SSHEs, light yellow regions to low concentration, and white to locations where there are no SSHEs.

background indicate that the bulk energy of the SSHEs ranges from $10^{21}$ erg to $10^{24} \mathrm{erg}$, while for a background of $1 \times$ $10^{17} \mathrm{erg} \mathrm{s}^{-1}$ the bulk energy of the SSHEs range from $10^{20} \mathrm{erg}$ to $10^{24} \mathrm{erg}$. The number of SSHEs increases rapidly with the decrease in the background cutoff. Therefore, it is reasonable to expect that the interval for the bulk energy of the SSHEs continues to increase with a decrease in the background value. Nevertheless, it is acceptable to assume that above the lower limit, considered to include the range of SSHEs that contribute enough energy to heat the corona, i.e., $1 \times 10^{18} \mathrm{erg}$ as explained in Paper I, the typical $E_{\mathrm{T}}$ of the SSHE ranges from $10^{20} \mathrm{erg}$ to $10^{23} \mathrm{erg}$. At the same time, the figure shows that the SSHEs that have a long lifetime are not the most energetic. In general, the most energetic SSHEs have lifetimes shorter than $150 \mathrm{~s}$. This suggests two types of SSHEs: those where most of the energy is released rapidly and those where the energy is released over longer periods of time. We note that for both models, a significant number of SSHEs with $E_{\mathrm{T}}$ above $10^{24} \mathrm{erg}$ are detected, where $10^{24}$ erg corresponds to the lower limit of the SSHEs that have already been detected observationally (Aschwanden et al. 2000). This opens up the possibility of comparing the properties of the simulated SSHEs with the observational properties of the SSHEs.

\subsection{Magnetic properties}

The conditions on the state of the magnetic field in order for reconnection to occur in the solar atmosphere are not fully understood. Parker (1983) argued that the magnetic field must be tangled or braided and that the tilt of the magnetic field in relation to the vertical opposing the direction of movement has to be on the order of $14^{\circ}$ for reconnection to occur. The secondary instability mechanism (Dahlburg et al. 1992), which is activated only when the magnetic field stress reaches a certain threshold, suggests that a critical angle of about $40^{\circ}$ has to be attained by the magnetic field rotating across the current sheets before reconnection occurs (Dahlburg et al. 2005).

The possibility of following the SSHEs over their lifetimes in the models allows us to calculate the directional change $(\alpha)$ of the field lines on the two sides of a current sheet. This gives us an idea of the magnetic field behavior when its energy is dissipated. The basic process by which current sheets are formed and reconnection initiates is slightly different in these numerical experiments from that envisioned by Parker (even though the expressions and estimates of the heating rate turn out to be roughly the same).

We estimate $\alpha$ over the lifetime of the SSHEs using the method described in Baumann et al. (2013). Essentially, the density of the electrical current in the current sheet $\left(j_{\mathrm{CS}}\right)$ is on the order of

$j_{\mathrm{CS}} \sim \Delta B \Delta s^{-1} \approx \sin (\alpha) B_{\mathrm{CS}} \Delta s^{-1} \approx \alpha B_{\mathrm{CS}} \Delta s^{-1}$.

In the case of small $\alpha, B_{\mathrm{CS}}$ stands for the typical strength of the magnetic field in the vicinity of the current sheet, $\alpha$ gives the directional change of the field lines on the two sides of the current sheet as described in Baumann et al. (2013) and $\Delta s$ is the thickness of the current sheet. As a first order approximation, $\alpha$ and $B_{\mathrm{CS}}$ are independent of $\Delta s$, thus we can write $\alpha \sim \Delta B / B_{\mathrm{CS}}$. However, $\Delta B$ is not straightforward to calculate; therefore, we 
estimate $\alpha$ as $\alpha \sim\left(j_{\mathrm{CS}} \Delta s\right) / B_{\mathrm{CS}}$. We assume the value of $\Delta s$ to be on the order of 2 and 4 grid cells in the LRM and HRM, respectively, so that the thickness of the current sheets is assumed to be roughly the same in both models for the purpose of these calculations. This assumption is more accurate in the regions where the magnetic field is nearly vertical because in this case $\Delta s=2 \Delta x$ or $\Delta s=4 \Delta x$ depending on the model, which is the same independent of the location in the simulation box.

We use $B_{\mathrm{CS}}=\left(B_{(x, y, z+1)}+B_{(x, y, z-1)}\right) / 2$ if the angle of the magnetic field with the vertical axis in the location of the local maximum of energy dissipation is greater than $45^{\circ}$ or $B_{\mathrm{CS}}=$ $\left(B_{(x+1, y, z)}+B_{(x-1, y, z)}\right) / 2$ if the angle of the magnetic field with the vertical in the location of the local maximum of energy dissipation is smaller than $45^{\circ}$. We calculated $\alpha$ for each instant of the lifetime of the SSHEs in the models. In the simulations, the angle is related to the topology of the magnetic field and the convective motions in the convective region. The angle we calculate measures the typical angle at which field lines intersect as photospheric motions attempt to force them to cross and pass through each other; that is, we do not find that reconnection is initiated as some critical angle is surpassed, rather the angle $\alpha$ is a measure of the typical relative tilt of field lines when they are pushed together, a scenario described earlier by Galsgaard \& Nordlund (1996) and confirmed here. Thus, this angle measures the efficiency of reconnection in pulling energy out of the magnetic field rather than measuring the critical angle at which reconnection occurs.

In particular, we calculated the value of $\alpha$ at $\max \left(\eta J_{\max }^{2}(t)\right)$ in the core cell of every SSHE and averaged it for each height. Figure 5 shows the average angle, $\langle\alpha\rangle$, against height for the LRM (top panel) and the HRM (bottom panel). The data was collected considering a background of $10^{17} \mathrm{erg} \mathrm{s}^{-1}$. In the LRM, $\langle\alpha\rangle$ ranges roughly between $1^{\circ}$ and $18^{\circ}$. The values of $\langle\alpha\rangle$ are small in the low atmosphere and increase toward the corona. In the upper atmospheric layers the magnetic field is nearly horizontal and $\Delta s=2 \Delta z$. Since the vertical dimension of the grid cells $(\Delta z)$ increases with height there is an overestimation of $\alpha$ in relation to the values calculated lower in the atmosphere. On the other hand, since the majority of the SSHEs occur in the vicinity of the footpoints and generally in this case $\Delta s=2 \Delta x$, which does not vary in the simulation box because the grid cells size do not change in the $x$-direction, we obtain a more accurate estimate for $\alpha$ from the SSHEs occurring in these locations. Therefore, the values of $\alpha$ can be estimated to range from $5^{\circ}$ to $15^{\circ}$, corresponding to a height interval ranging from 3 to $8 \mathrm{Mm}$. The error bars are given by the standard deviation $(\sigma)$ of $\alpha$ for each height.

In the HRM, $\alpha$ decreases from $14^{\circ}$ to roughly $5^{\circ}$ in the first $2 \mathrm{Mm}$. For some height points $\langle\alpha\rangle$ has relatively low values as seen at different heights, but above $2 \mathrm{Mm}\langle\alpha\rangle$ generally increases with height. The error bars are similar to those for the LRM and for clarity we do not display error bars for every data point, they are only shown for every tenth height point. In the upper atmosphere, as for the LRM there is an overestimation of the values of $\alpha$. Nevertheless, it is reasonable to assume that a typical value for $\alpha$ in this model ranges from $5^{\circ}$ to $15^{\circ}$ for heights from $2 \mathrm{Mm}$ to $6 \mathrm{Mm}$, assuming the same argument as for the LRM. We note that the $\langle\alpha\rangle$ range shown here can vary with the properties of the magnetic field in the different models. Although the two models have different magnetic properties, when we consider the SSHEs close to the footpoints and a similar thickness for the current sheets, the typical $\langle\alpha\rangle$ range is similar.
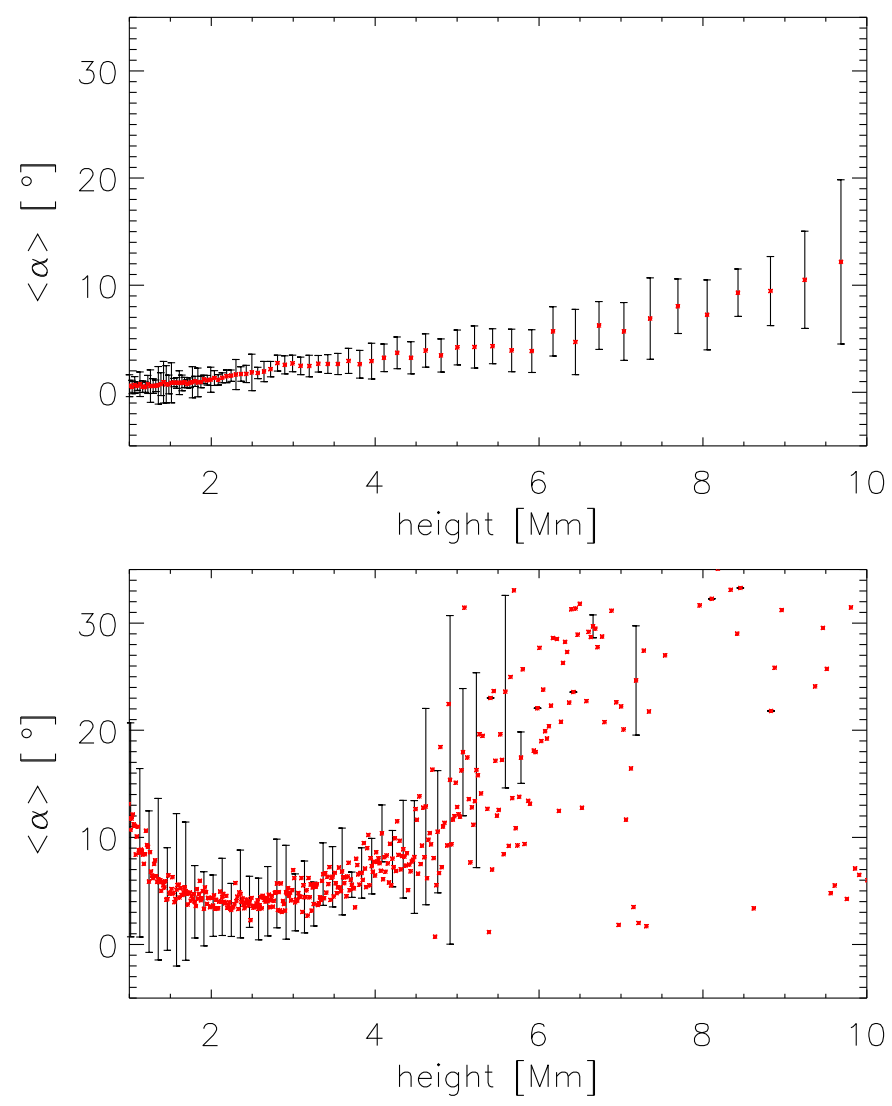

Fig. 5. Top panel: average directional change $(\langle\alpha\rangle)$ of the field lines at the moment of maximum dissipation in the core cell of the SSHEs during their lifetime $\left(\max \left(\eta J_{\max }^{2}(t)\right)\right)$ against height for the LRM. The data points correspond to the SSHEs computed with the background $10^{17} \mathrm{erg} \mathrm{s}^{-1}$. Bottom panel: same as the top panel, but for the HRM. In both cases the error bars correspond to the standard deviation $(\sigma)$ of $\alpha$ at each height.

\subsection{Detailed analyses of the plasma response to SSHES}

The method we developed allows for a detailed analysis of the different types of SSHEs that are tracked at different locations in the atmosphere and their properties. The SSHEs in these models generally do not move large distances during their lifetimes and they can be subdivided into two types: those that include violent reconnection and those that result from steady reconnection. These two types of SSHEs are responsible for maintaining the corona in the models presented here.

In Fig. 6, the properties and plasma response of two SSHEs over time with a cadence of $10 \mathrm{~s}$ are shown. These two SSHEs are identified as $\mathrm{SSHE}_{\mathrm{A}}$ and $\mathrm{SSHE}_{\mathrm{B}}$. In the figure, $\mathrm{SSHE}_{\mathrm{A}}$ is shown in the two left-hand columns and the $\mathrm{SSHE}_{\mathrm{B}}$ is shown in the two right-hand columns. The $\mathrm{SSHE}_{\mathrm{A}}$ was tracked in the LRM and $\mathrm{SSHE}_{\mathrm{B}}$ was tracked in the HRM; both SSHEs start occurring in regions corresponding to the upper transition region and lower corona. In both cases a background of $10^{17} \mathrm{erg} \mathrm{s}^{-1}$ was used.

For each SSHE, the figure displays the local maximum of the energy dissipation $\left(\eta J_{\max }^{2}\right)$ in a region (grid cell) inside the SSHE's volume. As mentioned before this is also the parameter used to track the SSHEs, the SSHE total energy $\left(E_{\mathrm{t}}\right)$ at each instant, and the directional change $(\alpha)$ of the field lines in the two sides of the current sheet in the local maximum dissipation region (cell). The figure also shows the density $(\rho)$, temperature 

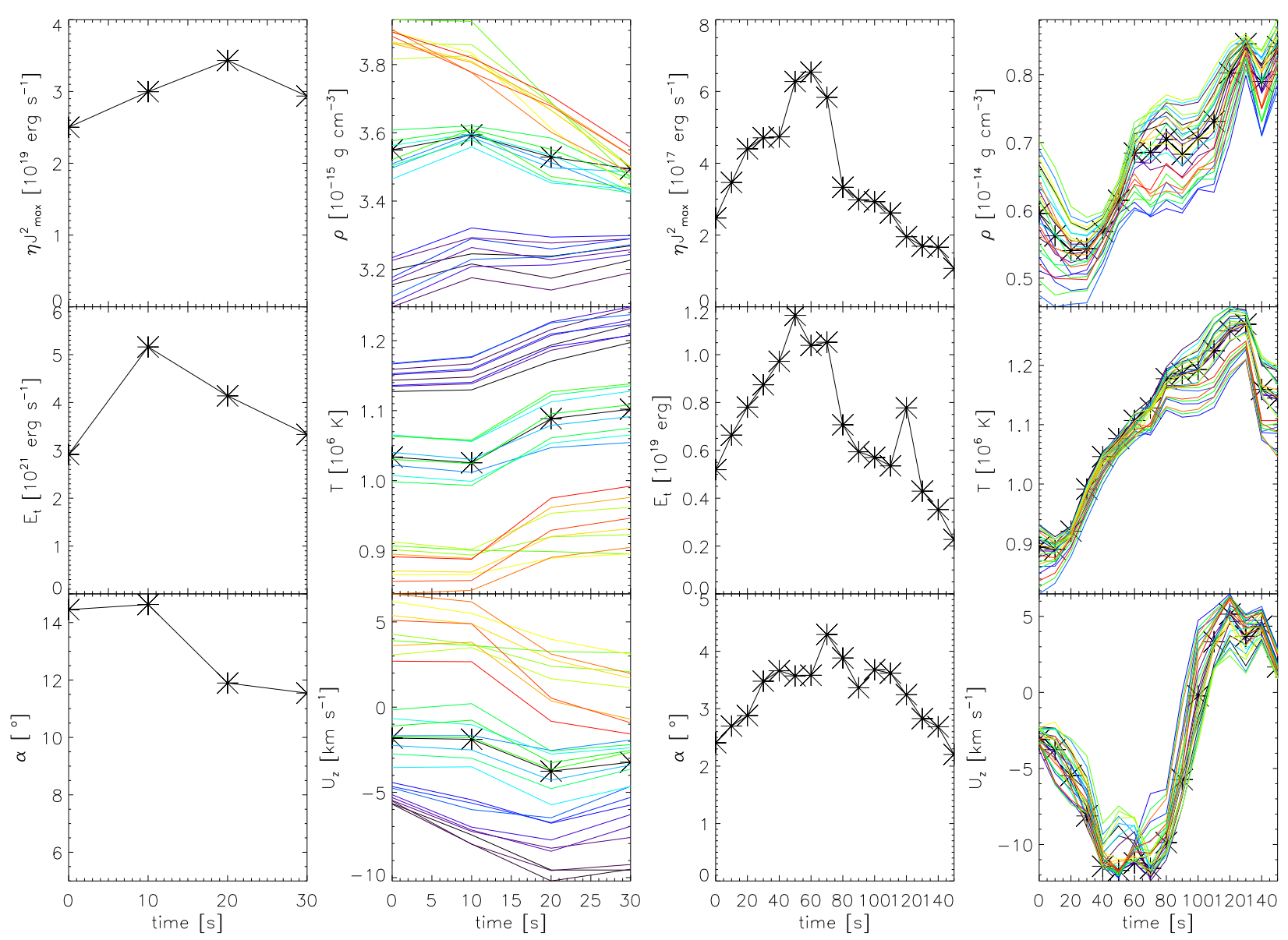

Fig. 6. Properties of two distinct SSHEs in the LRM and HRM over their lifetimes which start to occur in two different locations of the solar atmosphere. The properties of the $\mathrm{SSHE}_{\mathrm{A}}$ with a lifetime of $30 \mathrm{~s}$ are shown in the two left columns and the properties of the $\mathrm{SSHE}_{\mathrm{B}}$ with a lifetime of $150 \mathrm{~s}$ are shown in the two right columns. The asterisks in the first and third columns from the left give the energy dissipation $\left(\eta J_{\max }^{2}\right)$ at the maximum energy dissipation location of the SSHEs, the total energy $\left(E_{\mathrm{t}}\right)$ at each instant, and the directional change $(\alpha)$ of the magnetic field lines on the two sides of the current sheet in the local maximum dissipation region. The second and fourth columns from the left represent the density $(\rho)$, temperature $(T)$, and vertical component of the velocity $\left(U_{z}\right)$ in the vicinity of each SSHE's local maximum at each instant. The different colored lines represent the variables in the 27 cells that correspond to the core region of the SSHE over time. The asterisks in these groups of panels correspond to the value of the variables in the local maximum locations of the SSHEs every $10 \mathrm{~s}$ during their lifetime.

$(T)$, and vertical component of the plasma velocity $\left(U_{z}\right)$ in the vicinity of the region where $\eta J_{\max }^{2}$ occurs inside the volume of the SSHEs. The different colored lines correspond to the cells that have the same relative position in relation to the cell giving the $\eta J_{\max }^{2}$ irrespective of its displacement over time.

The $\mathrm{SSHE}_{\mathrm{A}}$ has a lifetime of $30 \mathrm{~s}$ and represents the SSHEs resulting from a violent reconnection process. The top left panel in the first column of the figure shows that the SSHE was detected when $\eta J_{\max }^{2}$ was $2.5 \times 10^{18} \mathrm{erg} \mathrm{s}^{-1}$ and we define this moment as the beginning of the lifetime of the $\mathrm{SSHE}_{\mathrm{A}}$, or $t=0 \mathrm{~s}$. We tracked the $\mathrm{SSHE}_{\mathrm{A}}$ during what we defined as the rising phase that lasted $t=20 \mathrm{~s}$ in this case, and $\eta J_{\max }^{2}$ reached a value of $3.4 \times 10^{18} \mathrm{erg} \mathrm{s}^{-1}$. The last $10 \mathrm{~s}$ of the lifetime of the $\mathrm{SSHE}_{\mathrm{A}}$ was the decaying phase where the value of $\eta J_{\max }^{2}$ decreased to $2.9 \times 10^{18} \mathrm{erg} \mathrm{s}^{-1}$ at the end of the lifetime of the $\mathrm{SSHE}_{\mathrm{A}}$, or $t=30 \mathrm{~s}$, which corresponds to the instant the $\mathrm{SSHE}_{\mathrm{A}}$ was lost by the algorithm. The middle panel in the first column gives the instantaneous total energy $\left(E_{\mathrm{t}}\right)$ of $\mathrm{SSHE}_{\mathrm{A}}$. The profile of $E_{\mathrm{t}}$ also shows a rising and decaying phase over the lifetime of the $\mathrm{SSHE}_{\mathrm{A}}$. However, the maximum occurs earlier than the maximum of $\eta J_{\max }^{2}$. At $t=0 \mathrm{~s}, E_{\mathrm{t}}=2.9 \times 10^{19} \mathrm{erg} \mathrm{s}^{-1}$ it then increases to its maximum of $5.1 \times 10^{19} \mathrm{erg} \mathrm{s}^{-1}$ at $t=10 \mathrm{~s}$. In the last $20 \mathrm{~s}, E_{\mathrm{t}}$ decreases to $3.3 \times 10^{19} \mathrm{erg} \mathrm{s}^{-1}$. The total energy dissipated by $\operatorname{SSHE}_{\mathrm{A}}\left(E_{\mathrm{T}}\right)$ over its lifetime is given by $\int_{0}^{30} E_{\mathrm{t}} \mathrm{d} t$, which is $E_{\mathrm{T}}=1.2 \times 10^{21} \mathrm{erg}$. The bottom left panel in the first column gives the directional change of the magnetic field $(\alpha)$. $\mathrm{SSHE}_{\mathrm{A}}$ was detected when the magnetic field already had a relatively large misalignment, which was $14.5^{\circ}$. The $\alpha$ increases to $14.6^{\circ}$ in the first $10 \mathrm{~s}$, and then decreases to $11.5^{\circ}$ at the end of the lifetime of the $\mathrm{SSHE}_{\mathrm{A}}$. The angle $\alpha$ increases until around the period that $E_{\mathrm{t}}$ is at a maximum. This is the moment when the violent reconnection occurs and after that, the value of $\alpha$ starts decreasing.

The density $(\rho)$, temperature $(T)$, and vertical component of the velocity $\left(U_{z}\right)$ are given in the second column from top to bottom, respectively. These panels give us information about how the plasma responds in the core region of the $\mathrm{SSHE}_{\mathrm{A}}$, where the core region is composed of 27 cells that surround and include the location where $\eta J_{\max }^{2}$ occurs. At the moment we started following the $\mathrm{SSHE}_{\mathrm{A}}$, the analyzed volume could be roughly subdivided into three regions, each of which has several cells: region $a_{1}$ given by the red, yellow, and light green lines; region $a_{2}$ given by the light green, turquoise, and the black lines; and region $a_{3}$ given by the purple and dark blue lines. In the volume composed of the 27 cells at $t=0 \mathrm{~s}$, the density of the $\mathrm{SSHE}_{\mathrm{A}}$ ranges from $3.0 \times 10^{-15} \mathrm{~g} \mathrm{~cm}^{-3}$ to $3.9 \times 10^{-15} \mathrm{~g} \mathrm{~cm}^{-3}$. In region $a_{1}$, 
some cells show a constant $\rho$ (light green lines) in the first $10 \mathrm{~s}$ and then decrease until the end of the lifetime of the $\mathrm{SSHE}_{\mathrm{A}}$. This region has the highest $\rho$ of the three regions and remains roughly at this value until the end of the lifetime of the $\mathrm{SSHE}_{\mathrm{A}}$. In the case of region $a_{2}$, the cells show an increase in $\rho$ in the first $10 \mathrm{~s}$ and then a decrease until the end of the lifetime of the $\mathrm{SSHE}_{\mathrm{A}}$. For region $a_{3}, \rho$ shows an increase in this region in the first $10 \mathrm{~s}$, then there is a decrease of $\rho$ until $t=20 \mathrm{~s}$, and then $\rho$ increases again in the last $10 \mathrm{~s}$. Region $a_{3}$ has the lowest $\rho$ during the lifetime of the $\mathrm{SSHE}_{\mathrm{A}}$. At the end of the lifetime, $\rho$ ranges from $3.1 \times 10^{-15}$ to $3.5 \times 10^{-15} \mathrm{~g} \mathrm{~cm}^{-3}$ in the core region of the $\mathrm{SSHE}_{\mathrm{A}}$. Generally, $\rho$ increases in the core region before $\alpha$ and $E_{t}$ reach their maxima. From this point on, $\rho$ shows a general decrease. This results most probably from the expansion of the material away from the region where the $\mathrm{SSHE}_{\mathrm{A}}$ is occurring. The temperature at $t=0 \mathrm{~s}$ in the core region of the $\mathrm{SSHE}_{\mathrm{A}}$ ranges from $8.3 \times 10^{5} \mathrm{~K}$ to $1.16 \times 10^{6} \mathrm{~K}$, and corresponds to a SSHE that starts occurring in the upper transition region and corona. In the first $10 \mathrm{~s}, T$ remains roughly constant in the three regions. After the maximum of $E_{t}, T$ increases in the three regions, roughly until the end of the lifetime, but the sharpest increase occurs from $t=10 \mathrm{~s}$ to $t=20 \mathrm{~s}$. At the end of the lifetime, $T$ ranges from $8.9 \times 10^{5} \mathrm{~K}$ to $1.24 \times 10^{6} \mathrm{~K}$. It is clear that $\mathrm{SSHE}_{\mathrm{A}}$ contributes to the increase in $T$ of the plasma in the neighborhood where it occurs. The vertical component of the plasma velocity $\left(U_{z}\right)$ ranges from $-6.6 \mathrm{~km} \mathrm{~s}^{-1}$ to $5.6 \mathrm{~km} \mathrm{~s}^{-1}$ at $t=0 \mathrm{~s}$. In regions $a_{1}$ and $a_{2}$ in the first $10 \mathrm{~s}, U_{z}$ remained roughly constant, from $t=10 \mathrm{~s}$ until $t=20 \mathrm{~s}$, region $a_{1}$ shows a decrease in $U_{z}$ that continues until the end of the lifetime of the $\mathrm{SSHE}_{\mathrm{A}}$, and region $a_{2}$ shows an increase in $U_{z}$ in absolute value, but it is reduced in the last $10 \mathrm{~s}$ of the lifetime. Region $a_{3}$ shows a decrease in $U_{z}$ in the first $20 \mathrm{~s}$ and an increase in the last $10 \mathrm{~s}$ of the lifetime of the $\mathrm{SSHE}_{\mathrm{A}}$. This indicates that the $\mathrm{SSHE}_{\mathrm{A}}$ caused an acceleration of the material downward at the same time as a deceleration of the material moving upward occurred.

The $\mathrm{SSHE}_{\mathrm{B}}$ has a lifetime of $150 \mathrm{~s}$, which is significantly longer-lived than the $\mathrm{SSHE}_{\mathrm{A}}$, that results from a steady reconnection process. The method detected $\mathrm{SSHE}_{\mathrm{B}}$ when $\eta J_{\max }^{2}$ was $2.47 \times 10^{17} \mathrm{erg} \mathrm{s}^{-1}$ (see third column from the left, top panel). We followed it during its rising phase until it reached a value of $6.54 \times 10^{17} \mathrm{erg} \mathrm{s}^{-1}$ at $t=60 \mathrm{~s}$. This is followed by a decaying phase until the end of the lifetime of the $\mathrm{SSHE}_{\mathrm{B}}$ at $t=150 \mathrm{~s}$, where $\eta J_{\max }^{2}=1.07 \times 10^{17} \mathrm{erg} \mathrm{s}^{-1}$. We detected the $\mathrm{SSHE}_{\mathrm{B}}$ when $E_{\mathrm{t}}=5.19 \times 10^{18} \mathrm{erg} \mathrm{s}^{-1}$ (see $3^{\text {rd }}$ column from the left, middle panel). As observed for $\eta J_{\max }^{2}, E_{\mathrm{t}}$ also follows a rising and decaying phase. It reaches a maximum value of $1.16 \times 10^{19} \mathrm{erg} \mathrm{s}^{-1}$ at $t=50 \mathrm{~s}$. During the decaying phase there are two instants were $E_{\mathrm{t}}$ increases and decreases subsequently at $t=70 \mathrm{~s}$ and $t=110 \mathrm{~s}$. These correspond to two larger bursts of energy, which we still consider part of the same SSHE. At the end of the lifetime, $E_{t}=2.27 \times 10^{18} \mathrm{erg}$. For $\mathrm{SSHE}_{\mathrm{B}}$, as was true for $\mathrm{SSHE}_{\mathrm{A}}$, the maximum of $E_{t}$ does not coincide with the maximum of $\eta J_{\max }^{2}$ and in this case $E_{T}$ occurs about $10 \mathrm{~s}$ before the maximum of $\eta J_{\max }^{2}$. The total energy dissipated, $E_{\mathrm{T}}$, for $\mathrm{SSHE}_{\mathrm{B}}$ is $\int_{0}^{150} E_{\mathrm{t}} \mathrm{d} t=1.0 \times 10^{21} \mathrm{erg}$, which is of the same order of magnitude as for $\mathrm{SSHE}_{\mathrm{A}}$, but results from a SSHE that is much smoother than $\mathrm{SSHE}_{\mathrm{A}}$. The $\alpha$ for the $\mathrm{SSHE}_{\mathrm{B}}$ is $\alpha=2.4^{\circ}$ at $t=0 \mathrm{~s}$ (see third column from the left, bottom panel). In the next $40 \mathrm{~s}, \alpha$ increases to $3.6^{\circ}$, and is followed by a decrease in $\alpha$ over the next $20 \mathrm{~s}$ where $\alpha=3.5^{\circ}$ at $t=60 \mathrm{~s}$. Thereafter, $\alpha$ increases to its maximum value of $4.3^{\circ}$ at $t=70 \mathrm{~s}$. This is followed by a decrease over the next $20 \mathrm{~s}$ and an increase such that at $t=100 \mathrm{~s}$ $\alpha=3.6^{\circ}$. From this point on, $\alpha$ decreases until the end of the lifetime of the SSHE where its value is $2.2^{\circ}$. This parameter is also characterized generically by a rising and decaying phase. In comparison to the $\mathrm{SSHE}_{\mathrm{A}}$, the variation of $\alpha$ is much smoother along the lifetime for $\mathrm{SSHE}_{\mathrm{B}}$ showing that $\mathrm{SSHE}_{B}$ results from a smoother reconnection process.

The density, temperature and vertical component of the velocity for $\mathrm{SSHE}_{\mathrm{B}}$ are given in the right-hand column of Fig. 6 from top to bottom, respectively. The values of $\rho$ in the cells that compose the core region of $\mathrm{SSHE}_{\mathrm{B}}$ present a general trend over the lifetime of the SSHE, i.e., in general when the density decreases or increases the same occurs in all cells. The $\rho$ at $t=0 \mathrm{~s}$ ranges from $4.75 \times 10^{-15} \mathrm{~g} \mathrm{~cm}^{-3}$ to $7.02 \times 10^{-15} \mathrm{~g} \mathrm{~cm}^{-3}$. The $\rho$ decreases in the first $20 \mathrm{~s}$ suggesting that during this time the material expands as a result of the $\mathrm{SSHE}_{\mathrm{B}}$. At this instant, $\rho$ ranges from $4.61 \times 10^{-15} \mathrm{~g} \mathrm{~cm}^{-3}$ to $6.06 \times 10^{-15} \mathrm{~g} \mathrm{~cm}^{-3}$. Thereafter, $\rho$ increases in general until $t=130 \mathrm{~s}$, where $\rho$ ranges from $7.98 \times 10^{-15} \mathrm{~g} \mathrm{~cm}^{-3}$ to $8.59 \times 10^{-15} \mathrm{~g} \mathrm{~cm}^{-3}$. In the next $10 \mathrm{~s}, \rho$ decreases generally and at $t=140 \mathrm{~s}$ it ranges from $7.09 \times 10^{-15} \mathrm{~g} \mathrm{~cm}^{-3}$ to $8.36 \times 10^{-15} \mathrm{~g} \mathrm{~cm}^{-3}$. In the last $10 \mathrm{~s} \rho$ shows an increase and at $t=150 \mathrm{~s}$ it ranges from $7.76 \times 10^{-15} \mathrm{~g} \mathrm{~cm}^{-3}$ to $8.80 \times 10^{-15} \mathrm{~g} \mathrm{~cm}^{-3}$. The increase in $\rho$ indicates that the expansion work resulting from the $\mathrm{SSHE}_{\mathrm{B}}$ is not sufficient to compensate for the compression caused by the plasma in the vicinity of the $\mathrm{SSHE}_{\mathrm{B}}$. The temperature in the core of the $\mathrm{SSHE}_{\mathrm{B}}$ ranges from $8.1 \times 10^{5} \mathrm{~K}$ to $9.3 \times 10^{5} \mathrm{~K}$ at $t=0 \mathrm{~s}$ corresponding to the upper transition region and in general $T$ increases until $t=120 \mathrm{~s}$, where $T$ ranges from $1.17 \times 10^{6} \mathrm{~K}$ to $1.3 \times 10^{6} \mathrm{~K}$. The $T$ in the core region decreases in the last $30 \mathrm{~s}$ to values that range from $1.1 \times 10^{6} \mathrm{~K}$ to $1.2 \times 10^{6} \mathrm{~K}$. It is also clear that despite being a smooth SSHE, it contributes significantly to an increase of the plasma temperature. The plasma in the core region of the $\mathrm{SSHE}_{\mathrm{B}}$ is moving downward at a rate ranging from $-2.24 \mathrm{~km} \mathrm{~s}^{-1}$ to $-3.80 \mathrm{~km} \mathrm{~s}^{-1}$. In the first $40 \mathrm{~s}$, the $\mathrm{SSHE}_{\mathrm{B}}$ contributes to the acceleration of the material downward. At around the moment the value of $E_{t}$ reaches a maximum, the $\mathrm{SSHE}_{\mathrm{B}}$ seems to contribute to the deceleration of the material moving downward until about $t=100 \mathrm{~s}$ where the plasma in the core region of the $\mathrm{SSHE}_{\mathrm{B}}$ starts accelerating upward. The plasma starts decelerating again roughly at $t=110 \mathrm{~s}$. Thereafter, $U_{z}$ decreases in general in the next $10 \mathrm{~s}$ followed by an increase. From $t=140 \mathrm{~s}$ until the end of the lifetime of the $\mathrm{SSHE}_{\mathrm{B}}, U_{z}$ decreases in general in the core region, except for the grid cells represented by the blue lines where the velocity is constant. At $t=150 \mathrm{~s}, U_{z}$ ranges from $0.91 \mathrm{~km} \mathrm{~s}^{-1}$ to $2.96 \mathrm{~km} \mathrm{~s}^{-1}$ in the core region of the $\mathrm{SSHE}_{\mathrm{B}}$. At this point in time, the $\mathrm{SSHE}_{\mathrm{B}}$ is in a location where the plasma is moving upward. This shows that the $\mathrm{SSHE}_{\mathrm{B}}$ can contribute to the reversion of the flow orientation over short periods of time. The $\mathrm{SSHE}_{\mathrm{B}}$ initially increases the acceleration of the plasma moving downward and at a later stage it contributes to a change in the orientation of the plasma movement.

\section{Conclusion}

In this work, we present a method for tracking SSHEs over their lifetimes in 3D-MHD models of the solar atmosphere with different spatial resolutions. Then, for the identified SSHEs, we determine their lifetimes, total energy, and magnetic properties together with plasma properties in their vicinity.

We applied the method to two models with different resolutions and magnetic topologies and selected the SSHEs using two different background limits, which were $10^{17} \mathrm{erg} \mathrm{s}^{-1}$ and $10^{18} \mathrm{erg} \mathrm{s}^{-1}$. We found that the SSHEs studied in the models at the resolution presented here have an average lifetime that ranges 
from 50 to $60 \mathrm{~s}$. There is a small difference in the average lifetime of the SSHEs in the different models, but the results fall well inside the uncertainty of both models. The variation of the background has a marginal impact on the SSHE's lifetime. The study of SSHEs in similar models by Hansteen et al. (2015) using a different method yielded similar results for the average lifetime of the SSHEs. It is difficult to argue that the average lifetimes in the models are fully representative of the lifetimes of the SSHEs that heat the corona, since the very short-lived SSHEs could be very relevant and we cannot account for them at the moment because of the limited temporal resolution of the model. The models would need a higher temporal resolution to be able to properly assess the role of SSHEs with a very short lifetime in coronal heating. On the other hand, the analyses of the models give us a general upper limit for the lifetime of the SSHEs that heat the corona which is on the order of $4 \mathrm{~min}$.

The majority of the SSHEs in the two models have a total energy that ranges roughly from $10^{20} \mathrm{erg}$ to $10^{24} \mathrm{erg}$. As shown in Paper I, the SSHEs that have a total energy greater than $10^{21} \mathrm{erg}$ in the LRM can provide enough energy to sustain the corona, thus it is reasonable to assume that the properties of the SSHEs we present here are representative of those that heat the corona. However, we will only have a complete picture of the SSHEs that heat the upper layer of the solar atmosphere when we are able to analyze the SSHEs that have very short lifetimes, which also have the potential to release large amounts of energy. It is also worth noting that generally the most energetic SSHEs have a short lifetime, but there is no direct correlation between the length of the lifetime and the total energy dissipated $\left(E_{\mathrm{T}}\right)$ by the SSHEs.

The directional change, $\alpha$, of the field lines from one side of the current sheet to the other over the lifetime of the SSHEs is similar in the two models when we consider the current sheets to have roughly the same thickness. In the two models, the current sheet thickness can be compared in the footpoint region. At the instant that $\eta J_{\max }^{2}(t)$ is at a maximum, $\alpha$ ranges roughly from $5^{\circ}$ to $15^{\circ}$ in that region. This indicates that reconnection extracts energy most efficiently from the magnetic field in this $\alpha$ range.

We analyzed in detail the properties of two SSHEs. These essentially represent two types of SSHEs that are found in the models. The SSHEs that dissipate energy through a violent reconnection process which in general are shorter-lived and those that live longer, are much smoother and the energy is dissipated through steady reconnection. Independent of the type of the SSHEs, in general they do not move long distances (or over many cells) over their lifetimes. The increase in the temperature can occur in a progressive way when the SSHEs start occurring; in some cases it starts after the $E_{\mathrm{t}}$ reaches its maximum, which in general corresponds to the moment the when violent reconnection occurs.

The density generally shows a small decrease at the beginning of the SSHEs resulting from the expansion of the plasma at the location where the SSHEs start to occur. After the initial phase, it is difficult to establish the behavior of the density; sometimes it increases and sometimes it decreases as the SSHEs evolve. However, toward the end of the lifetime of the SSHEs, $\rho$ generally increases, due to the decrease in the SSHEs that reduce the plasma pressure. The effect of the SSHEs in the upward and downward plasma flow is not straightforward. In some cases, we see a clear acceleration of the plasma, while in other cases deceleration occurs. The acceleration or deceleration might be related to the location in the atmosphere where the SSHEs occur. A further analysis of the properties of the plasma and the energy dissipated is needed to better understand what drives the acceleration or deceleration of the plasma as a result of a SSHE.

We cannot currently fully study the influence of the model resolution on the lifetime of the SSHEs and we cannot include all the microphysics necessary to reproduce many of the complex phenomena in detail that occur at small scales in the solar atmosphere. The former occurs because we currently do not have the same model with two resolutions spanning the same time period, while the latter is currently unattainable due to the prohibitive computing power that such simulations would require. Despite these challenges, the method presented here can significantly contribute to a better understanding of SSHEs and their influence on the plasma in their vicinity. The method will also facilitate the study of several other aspects in the future, for example the energy balance of the SSHEs, their spectral signatures, and understanding if the SSHEs cluster by chance or in an avalanche-like process, among other possibilities. Also, the question of whether the occurrence rate of the SSHEs shows a dependence on the solar activity, i.e., the magnetic field strength, is yet to be addressed. Ultimately, SSHEs could be the main driver of the solar cycle variation in coronal emission (see, e.g., Haberreiter et al. 2014). Finally, this work is extremely relevant for the interpretation, and the potential observational evidence of the SSHEs with respect to the upcoming data from the Spectral Imaging of the Coronal Environment instrument (SPICE; Fludra et al. 2013) and the Extreme UV imager (EUI; Halain et al. 2014) on board the Solar Orbiter Mission.

Acknowledgements. N.G. is greatful for the financial support from the Swiss National Science Foundation through grant No. 153302. He would also like to thank S. Nyeki and T. Solms for relevant contributions and the referee for important comments that significantly improved the paper. M.H. acknowledges funding from the European Community's Seventh Framework Programme (FP7 2012) under Grant Agreement No. 313188 (SOLID, http://projects.pmodwrc. ch/solid).

\section{References}

Achour, H., Brekke, P., Kjeldseth-Moe, O., \& Maltby, P. 1995, ApJ, 453, 945 Aschwanden, M. J., Nightingale, R. W., Tarbell, T. D., \& Wolfson, C. J. 2000, ApJ, 535, 1027

Aschwanden, M. J., Crosby, N. B., Dimitropoulou, M., et al. 2016, Space Sci. Rev. 298, 47

Baumann, G., Galsgaard, K., \& Nordlund, Å. 2013, Sol. Phys., 284, 467 Berghmans, D. 2002, in Solar Variability: From Core to Outer Frontiers, ed. A. Wilson, ESA SP, 506, 501

Berghmans, D., Clette, F., \& Moses, D. 1998, A\&A, 336, 1039

Bingert, S., \& Peter, H. 2011, A\&A, 530, A112

Bingert, S., \& Peter, H. 2013, A\&A, 550, A30

Bowness, R., Hood, A. W., \& Parnell, C. E. 2013, ApJ, 560, A89

Brekke, P. 1993, ApJ, 408, 735

Brekke, P., Hassler, D. M., \& Wilhelm, K. 1997, Sol. Phys., 175, 349

Browning, P. K., Gerrard, C., Hood, A. W., Kevis, R., \& van der Linden, R. A. M. 2008, A\&A, 485, 837

Cargill, P. J. 1994, ApJ, 422, 381

Cargill, P. J., \& Klimchuk, J. A. 2004, ApJ, 605, 911

Carlsson, M., \& Leenaarts, J. 2012, A\&A, 539, A39

Dahlburg, R. B., Antiochos, S. K., \& Zang, T. A. 1992, Phys. Fluids B, 4, 3902 Dahlburg, R. B., Klimchuk, J. A., \& Antiochos, S. K. 2005, ApJ, 622, 1191

De Pontieu, B., McIntosh, S., Hansteen, V. H., et al. 2007, PASJ, 59, 655

Dere, K. P. 1982, Sol. Phys., 77, 77

Doschek, G. A., Bohlin, J. D., \& Feldman, U. 1976, ApJ, 205, L177

Fludra, A., Griffin, D., Caldwell, M., et al. 2013, SPIE Conf. Ser., 8862

Galsgaard, K., \& Nordlund, A. 1996, J. Geophys. Res., 101, 13445

Gebbie, K. B., Hill, F., November, L. J., et al. 1981, ApJ, 251, L115

Gold, T. 1964, NASA Spec. Publ., 50, 389

Gudiksen, B. V., Carlsson, M., Hansteen, V. H., et al. 2011, A\&A, 531, A154

Gudiksen, B. V., \& Nordlund, Å. 2005, ApJ, 618, 1020

Guerreiro, N., Carlsson, M., \& Hansteen, V. 2013a, ApJ, 766, 128

Guerreiro, N., Hansteen, V., \& De Pontieu, B. 2013b, ApJ, 769, 47 
N. Guerreiro et al.: Small-scale heating events in the solar atmosphere. II.

Guerreiro, N., Haberreiter, M., Hansteen, V., \& Schmutz, W. 2015, ApJ, 813, 61 Haberreiter, M., Delouille, V., Mampaey, B., et al. 2014, J. Space Weather Space Clim., 4, A30

Halain, J.-P., Rochus, P., Renotte, E., et al. 2014, SPIE Conf. Ser., 9144, 8

Hannah, I. G., Christe, S., Krucker, S., et al. 2008, ApJ, 677, 704

Hansteen, V. 1993, ApJ, 402, 741

Hansteen, V. H., Carlsson, M., \& Gudiksen, B. 2007, in The Physics of Chromospheric Plasmas, eds. P. Heinzel, I. Dorotovič, \& R. J. Rutten, ASP Conf. Ser., 368, 107

Hansteen, V. H., Hara, H., De Pontieu, B., \& Carlsson, M. 2010, ApJ, 718, 1070

Hansteen, V., Guerreiro, N., De Pontieu, B., \& Carlsson, M. 2015, ApJ, 811, 106

Hayek, W., Asplund, M., Carlsson, M., et al. 2010, A\&A, 517, A49

Hendrix, D. L., van Hoven, G., Mikic, Z., \& Schnack, D. D. 1996, ApJ, 470, 1192

Klimchuk, J. A. 1987, ApJ, 323, 368

Klimchuk, J. A. 2006, Sol. Phys., 234, 41

Klimchuk, J. A. 2015, Roy. Soc. London Philos. Trans. Ser. A, 373, 40256

Klimchuk, J. A., \& Cargill, P. J. 2001, ApJ, 553, 440

Krucker, S., \& Benz, A. O. 1998, ApJ, 501, L213

Lin, R. P., Schwartz, R. A., Kane, S. R., Pelling, R. M., \& Hurley, K. C. 1984, ApJ, 283, 421
Nordlund, A. 1982, A\&A, 107, 1

Parker, E. N. 1972, ApJ, 174, 499

Parker, E. N. 1983, ApJ, 264, 642

Parker, E. N. 1988, ApJ, 330, 474

Parnell, C. E., \& De Moortel, I. 2012, Roy. Soc. London Philos. Trans. Ser. A, 370,3217

Parnell, C. E., \& Jupp, P. E. 2000, ApJ, 529, 554

Peter, H., \& Judge, P. G. 1999, ApJ, 522, 1148

Porter, J. G., Toomre, J., \& Gebbie, K. B. 1984, ApJ, 283, 879

Porter, J. G., Moore, R. L., Reichmann, E. J., Engvold, O., \& Harvey, K. L. 1987, ApJ, 323, 380

Priest, E. R., Heyvaerts, J. F., \& Title, A. M. 2002, ApJ, 576, 533

Ramesh, R., Sasikumar Raja, K., Kathiravan, C., \& Narayanan, A. S. 2013, ApJ, 762,89

Reale, F. 2010, Liv. Rev. Sol. Phys., 7, 5

Rottman, G. J., Hassler, D. D., Jones, M. D., \& Orrall, F. Q. 1990, ApJ, 358, 693

Steffen, M., \& Muchmore, D. 1988, A\& A, 193, 281

Sterling, A. C. 2000, Sol. Phys., 196, 79

Tomczyk, S., McIntosh, S. W., Keil, S. L., et al. 2007, Science, 317, 1192

Wang, T. J., Innes, D. E., Solanki, S. K., \& Curdt, W. 2015, ArXiv e-prints [arXiv: 1510.00337] 